\title{
NUMERICAL MODELLING OF SHOCK WAVES IN THE SOLAR WIND
}

\author{
Y. Q. HU \\ University of Science and Technology of China, \\ Hefei, Anhui 230026, PRC
}

July 1,1996

\begin{abstract}
This review briefly surveys some recent progress in the numerical simulation study of magnetohydrodynamic (MHD) shock waves in the solar wind.
\end{abstract}

\section{STANDING SHOCKS IN STEADY SOLAR WIND}

In order to accelerate the solar wind it is necessary to add energy or momentum to it. The energy or momentum addition creates more than one critical points, resulting in discontinuous solar wind solutions involving standing shocks [Holzer, 1977]. In terms of an isothermal model, Habbal and Tsinganos [1983] showed that multiple transonic solutions may exist for a given set of coronal base parameters owing to the presence of multiple critical points. One of these solutions is continuous while the others are all discontinuous, containing one or more standing shocks. Habbal and Rosner [1984] further examined the temporal evolution of an isothermal solar wind from one steady state to another under the action of momentum addition, and concluded that the pattern of the final steady state depends on the amplitude and the rate of momentum addition. This conclusion also holds for the polytropic solar wind [Habbal 1985]. Using a two-fluid model, Habbal et al. [1994] found a solar wind solution with two standing shocks. These studies indicate that solar winds with standing shocks are not only allowed by the steady state equations, but also physically accessible and stable. The presence of standing shocks bring about a remarkable change of the inner solar wind structure, which could be observationally detected [Esser and Habbal, 1990]. The transversal nonuniformity of the interplanetary magnetic field provides another physical basis for the formation of standing shocks. Whang $[1982,1986]$ suggested that a slow shock develops at the inner edge of the heliospheric current sheet and extends polewards to form a close surface around the Sun, and showed a typical solar wind solution with the close slow shock. If the prediction of discontinuous solar winds turns out to be confirmed by future observations, it will certainly bring about a revolution in the solar wind theory.

A great upsurge in the study of the heliospheric boundary started several years ago and has since maintained since one or more of the deep space 
probes Voyagers $1 \& 2$ and Pioneers $10 \& 11$ are expected to yield in situ observations of this boundary region. The boundary may consist of a termination shock, an interface (heliopause), and probably a bow shock. Tentative simulations of the solar wind interaction with the local interstellar medium were carried out based on gas dynamic models [Baranov and Malama, 1993; Steinolfson, 1994] although the local interstellar conditions are poorly known. Various investigations came to a common conclusion: the nearest heliocentric distance of the termination shock is in the range of 70 $100 \mathrm{AU}$ and keeps changing in response to solar wind fluctuations with a speed greater than or comparable to the speed of the spacecraft [Suess, 1993; Cummings et al., 1993; Belcher et al., 1993; Whang and Burlaga, 1993].

\section{INTERPLANETARY COROTATING SHOCK WAVES}

Observations show that corotating shocks start to form near $1 \mathrm{AU}$, developing rapidly then, and the pressure ratio may reach a few thousands at 4-5 AU [Whang and Burlaga, 1988]. The reverse shock forms earlier, but eventually becomes weaker than its forward counterpart outside a certain heliocentric distance. Corotating shocks are weak or absent at 1 AU. Gosling et al. [1978] collected 28 high-speed stream events and found only three in them accompanied by corotating shocks which were all reverse ones.

Under the perpendicular shock approximation and using spacecraft observations, Whang made a successful study of the formation, propagation and interaction of corotating shocks in the outer heliosphere, which dominates the dynamics as well as the heating of the outer heliosphere [see Whang, 1991a]. $\mathrm{Hu}$ and Habbal [1993a] made a general analysis of the interaction between perpendicular shocks. Pizzo [1982] and Whang and Chien [1981] adopted multi-dimensional steady models to study high-speed streams. Recently, Pizzo [1994] carried his three-dimensional steady MHD simulations up to $50 \mathrm{AU}$ with emphasis on the origin of north-south flows and the deformation of the heliospheric current sheet, but the corotating shocks obtained suffered from a high level of numerical diffusion. Hu [1993] developed an effective algorithm and applied it to simulation studies of two typical highspeed streams in the heliocentric equatorial plane from $0.3 \mathrm{AU}$ up to $10 \mathrm{AU}$, depicting a complete picture of the formation, development and interaction of corotating shocks.

\section{SHOCK WAVES ASSOCIATED WITH CORONAL MASS EJECTIONS (CMEs)}

Hundhausen et al. [1987] made the suggestion that slow shocks may form as the CMEs sweep through the corona. To quantify such a suggestion, $\mathrm{Hu}$ 
et al. [1989] used a 2-D MHD model in spherical coordinates to study the formation of the slow shock associated with CMEs. The shock obtained is characterized by a limited transversal extent and a concave- upward shape, as well as a coexisting fast magnetoacoustic wave. Later on, Steinolfson and Hundhausen [1989] made a similar simulation study in Cartesian coordinates and reached essentially identical conclusion. The slow shock - fast magnetoacoustic wave system keeps evolving during its propagation outward, resulting in a hybrid shock consisting of an intermediate and a fast shock [Hu et al., 1992; Steinolfson and Hundhausen 1990a, 1990b].

\section{TRAVELLING SHOCKS ASSOCIATED WITH SOLAR DISTURBANCES}

It was recognized as early as 1970 s that the propagation of the flare-shock is asymmetrical relative to the flare normal, a radial ray passing through the flare. Sakurai and Chao [1973] and Watanabe [1977] argued that the shock propagation speed takes its maximum along the interplanetary spiral field line. Zhang [1984] made a statistical analysis of the relationship between solar flares and heliospheric and geomagnetic disturbances and further confirmed the existence of the east-west asymmetry. Wei [1987] and Wei and Dryer [1991] concluded that the fastest propagation direction of the flareshock tends towards the spiral magnetic field in longitudes and approaches the heliospheric electric current sheet in latitudes, and that high dynamical parameters and weak magnetic field appear in the south-east part of the shock whereas the opposite is true for the north-west. However, previous simulation studies produced shock fronts almost symmetrical relative to flare normal [Smith and Dryer, 1987]. This indicates that whether the shock speed is symmetrical to the flare normal remains in dispute and merits further exploration.

Disturbances varying gently with time and space steepen through nonlinear evolution which provides another common source for interplanetary shocks. $\mathrm{Hu}$ and Habbal [1993b] found that a pure velocity enhancement disturbance in the inner solar wind evolves into a double shock pair, with a forward- reverse slow shock pair sandwiched between the ordinary pair of fast shocks. Whang [1991b, 1991c] presented a parametric study of the formation and evolution of MHD shocks in the inner heliosphere; a slow shock pair starting from the near Sun region was found evolving into a fast shock pair before its arrival at the Earth. $\mathrm{Hu}$ [1994] studied the evolution of a single forward slow shock in the interplanetary space and found that it diminishes by launching fast compression waves and evolves gradually into a quasi-tangential discontinuity, and the launched fast compression waves 
transform into a fast shock ahead of the original slow shock through nonlinear steepening.

Since intermediate shocks may form by steepening of continuous waves and exist stably, as proved by $\mathrm{Wu}$ [1990], one will naturally think of the possible existence of such shocks in the interplanetary space. Some conjectures have been put forward on the existence of intermediate shocks as well as hybrid shocks associated with them in the interplanetary space $[\mathrm{Hu}, 1991 \mathrm{a}$, 1992]. Watanabe et al. $[1988,1989]$ found for the first time that the shock front is concave towards the Sun in the vicinity of the heliospheric electric current sheet. $\mathrm{Hu}$ [1991b] argued that the shock observed by them is probably a hybrid shock, its central part being an intermediate one concave to the Sun. Recently, from the plasma and magnetic field data of Voyager 1 Chao et al. [1993] identified an intermediate shock of $2 \rightarrow 4$ type at a distance about $9 \mathrm{AU}$ and $0.2 \mathrm{AU}$ above the ecliptic plane.

In terms of a coplanar shock-tube problem, Brio and Wu [1988] found for the first time that a compound wave may form due to the nonconvexity of the MHD equations, consisting of a shock and a rarefaction wave of the same mode. Compound waves may occur in fast magnetic reconnection regions. Ugai [1993] studied the fast reconnection in a sheared field geometry and obtained a switch-off shock accompanied by a stationary intermediate wave.

\section{NUMERICAL TECHNIQUES IN THE TREATMENT OF SHOCKS}

For flows containing shocks a careful treatment of shocks is of vital importance to the accuracy of the numerical results. Various numerical techniques have been developed for shock treatment, and in what follows, we list a few of them that were successfully applied to numerical simulations for space shocks.

Numerical and Artificial Dissipation Method uses numerical schemes of strong numerical dissipation or adds artificial dissipation terms to the equations so as to prevent numerical instabilities and oscillations on both sides of the shock. Such a technique has been adopted by most simulation researchers because of simplicity in programming and low cost of computer time. A shock is smeared out over 4-5 meshes. Flux-Corrected-Transport (FCT) it Method first adds a sufficient positive dissipation to the numerical solution so as to suppress instabilities associated with shocks, and then exerts an adequate negative dissipation to compensate for numerical errors introduced by the positive dissipation so as to raise the accuracy and the resolution of shock calculation. The FCT method was first developed by Boris and Book [Boris and Book, 1973; Book et al., 1975; Boris and Book, 1976] and later on gen- 
eralized by Zalesak [1979]. With the FCT method, the shock layer thickness may reduce to 2-3 spatial meshes. Adaptive Grid Method adjusts the layout of grid points at all times so as to maintain a higher density of grid points in the shock region and consequently, to improve the accuracy and the resolution of shock calculation [Wu et al., 1987]. Piecewise Parabolic Method (PPM) uses a parabolic function to improve the accuracy of interpolation in space, allowing for a sharp representation of shocks, and includes an effective Riemann solver to handle the interaction between nonlinear waves within shock layers so as to correct the fluxes at the grid points there. It may resolve a shock to 1-2 meshes [see Colella and Woodward, 1984; Zachary and Colella, 1992; Zachary et al., 1993]. The above-mentioned approaches all belong to the category of "shock capturing", in which shocks are smeared out more or less by numerical diffusion on the grid. Woodward and Collella [1984] made an extensive comparison between various shock capturing methods. According to their analysis, a more sophisticated scheme like PPM achieves a higher accuracy per zone of a uniform grid, but needs more computer time. If one measures accuracy per CPU time, there is a significantly smaller spread between various schemes than if one measures accuracy per zone of the grid. Therefore, Woodward and Collella suspected that all of their research may eventually lead to a conservation of difficulty principle. For very strong shocks in the outer heliosphere with fast magnetoacoustic Mach numbers of 10 and greater or pressure ratios of $10^{2}$ and greater, the shock capturing method can not attain sufficient entropy increase across shocks. Shock Fitting Method treats a shock as a discontinuity of zero thickness and uses the Rankine-Hugoniot relations to match the flow conditions on the two sides of the shock. The main procedure is to divide the entire domain of solution into several continuous flow regions bordered by the shock surfaces, to obtain the solutions in these regions, and to match these solutions in terms of Rankine-Hugoniot relations. Whang was the first to apply the shock fitting method to study 1-D, time-dependent perpendicular [Whang, 1984] and oblique [Whang, 1991b] shocks in the solar wind in terms of a characteristic grid. Recently, such method was extended to an Eulerian grid for 1-D, time- dependent oblique shocks [Hu and Habbal, 1993b] and 2-D, steady shocks [Hu, 1993]. Their simulations indicate that the shock fitting method appears a most promising method in handling space shocks.

\section{References}

Baramov, V. B. and Malama, Y. G., 1993 J. Geophys. Res., 98, 15157

Belcher, J. W., Lazarus, A. J., McNutt, Jr. R. L., and Gordon, Jr. G. S., 1993, J. Geophys. Res., 98, 15177

Book, D. L., Boris, J. P., and Hain, K., 1975, J. Comput. Phys., 18, 248

Boris, J. P. and Book, D. L., 1973, J. Comput. Phys., 11, 38

Boris, J. P. and Book, D. L., 1976, J. Comput. Phys., 20, 397 
Brio, M. and Wu, C. C., 1988, J. Comput. Phys., 75, 400

Chao, J. K., Lyu, L. H., Wu, B. H., Lazarus, A. J., and Chang, T. S., 1993, J. Geophys. Res., 98, 17443

Colella, P., and Woodward, P. R., 1984, J. Comput. Phys., 54, 174

Cummings, A. C., Stone, E. C., and Webber, W. R., 1993, J. Geophys. Res., 98, 15165

Esser, R. and Habbal, S. R., 1990, Solar Phys., 129, 153

Gosling, J. T., Asbridge, J. R., Bame, S. J., and Feldman, W. C., 1978, J. Geophys. Res., 83, 1401

Habbal, S. R., 1985, J. Geophys. Res., 90, 199

Habbal, S. R., Hu, Y. Q., and Esser, R., 1994, J. Geophys. Res., 99,

Habbal, S. R., and Rosner, R., 1984, J. Geophys. Res., 89, 10645

Habbal, S. R., and Tsinganos, 1983, J. Geophys. Res., 88, 1965

Holzer, T. E., 1977, J. Geophys. Res., 82, 23

Hu, Y. Q., 1991a, J. Geomag. Geoelectr., 43 Suppl., 11

Hu, Y. Q., 1991b, Acta Geophysica Sinica, 34, 397

Hu, Y. Q.: 1992, " in W. R. Hu, B. R. Zhang, and D. R. Lu, ed(s)., in Advances in Solar-Terrestrial Science of China, Science Press: Beijing, New York, p.96

Hu, Y. Q., 1993, J. Geophys. Res., 98, 1320

Hu, Y. Q., 1994, Acta Geophysica Sinica, 37, 257

Hu, Y. Q. and Habbal, S. R., 1993a, Phys. Fluids B, 5, 732

Hu, Y. Q. and Habbal, S. R., 1993b, J. Geophys. Res., 98, 3551

Hu, Y. Q., Zhu, Z. W., Hundhausen, A. J., Holzer, T. E., and Low, B. C., 1989, Science in China Ser. A, No.11, 1178

Hu, Y. Q., Wang, C., and Zheng, H. N., 1992, Acta Astrophysica Sinica, 12, 54

Hundhausen, A. J., Holzer, T. E., and Low, B. C., 1987, J. Geophys. Res., 92, 11173

Pizzo, V. J., 1982, J. Geophys. Res., 87, 4374

Pizzo, V. J., 1994, J. Geophys. Res., 99, 4173-4192

Sakurai, K., and Chao, J. K., 1973, Nature Phys. Sci., 246, 72

Smith, Z. and Dryer, M.: 1987, " in V.J. Pizzo et al., ed(s)., in Proceedings of the Sixth International Solar Wind Conference, HAO/NCAR, p.309

Steinolfson, R. S., 1994, J. Geophys. Res., 99, 13307

Steinolfson, R. S. and Hundhausen, A. J., 1989, J. Geophys. Res., 94, 1222

Steinolfson, R. S. and Hundhausen, A. J., 1990a, J. Geophys. Res., 95, 6389

Steinolfson, R. S. and Hundhausen, A. J., 1990b, J. Geophys. Res., 95, 20693

Steinolfson, R. S., Dryer, M., and Nakagawa, Y., 1975, J. Geophys. Res., 80, 1223

Suess, S. T., 1993, J. Geophys. Res., 98, 15147

Ugai, M., 1993, Phys. Fluids B, 5, 3021

Watanabe, T.: 1977, ' in M. A. Shea et al., ed(s)., in The Study of Travelling Interplanetary Phenomena, D.Reidel Pub. Co.: Dordrecht-Holland/Boston-USA, p.139

Watanabe, T., Kakinuma, T., and Kojima, M.: 1988, " in K. Kikoch, ed(s)., in Laboratory and Space Plasmas, Springer-Verlag: Berlin, p.399

Watanabe, T., Kakinuma, T., Kojima, M., and Schwenn, R., 1989, Proc. Res. Inst. Atmospherics, Nagoya Uni., 36, 11

Wei, F. S., 1987, Scientia Sinica Ser. A, No.2, 186

Wei, F. S. and Dryer, M., 1991, Solar Phys., 132, 373

Whang, Y. C., 1982, Geophys. Res. Lett., 9, 1081

Whang, Y. C., 1984, J. Geophys. Res., 89, 7367

Whang, Y. C., 1986, Astrophys. J., 307, 838

Whang, Y. C., 1991a, Space Sci. Rev., 57, 339

Whang, Y. C., 1991b, Astrophys. J., 377, 250-262

Whang, Y. C., 1991c, Astrophys. J., 381, 559

Whang, Y. C. and Burlaga, L. F., 1988, J. Geophys. Res., 93, 5446

Whang, Y. C. and Burlaga, L. F., 1993, J. Geophys. Res., 98, 15221

Whang, Y. C. and Chien, T. H., 1981, J. Geophys. Res., 86, 3263

Woodward, P. and Collela, P., 1984, J. Comput. Phys., 54, 115 
Wu, C. C., 1990, J. Geophys. Res., 95, 8149

Wu, S. T., Panitchob, S., and Suess, S.T.: 1987, " in V.J. Pizzo et al., ed(s)., in Proc. of the Sixth International Solar Wind Conf., HAO/NCAR, p.303

Zachary, A. L. and Colella, P., 1992, J. Comput. Phys., 99, 341

Zachary, A. L., Malagoli, A., and Collela, P., 1993, SIAM J. Scientific and Statistical Computing, (in press)

Zalesak, S. T., 1979, J. Comput. Phys., 31, 335

Zhang, G. L., 1984, Scientia Sinica Ser. A, No.3, 254 